\title{
UAV Aided Network Association in Space-Air-Ground Communication Networks
}

\author{
Jingjing Wang*, Chunxiao Jiang*, Zhongxiang $\mathrm{Wei}^{\dagger}$, Tong $\mathrm{Bai}^{\ddagger}$, Haijun Zhang ${ }^{\S}$ and Yong Ren* \\ *Tsinghua University, Beijing, 100084, China \\ ${ }^{\dagger}$ University College London, London, WC1E 6BT, United Kingdom

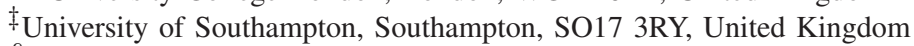 \\ $\S$ University of Science and Technology Beijing, Beijing, 100083, China \\ Email: chinaeephd@gmail.com
}

\begin{abstract}
Equipped with diverse communication payloads, unmanned aerial vehicles (UAVs) cooperating with satellites and base stations (BSs) constitute a space-air-ground three-tier heterogeneous network, which are beneficial in terms of both providing the seamless coverage as well as of improving the capacity for the users. However, cross-tier interference may be inevitable among these tightly embraced heterogeneous networks. In our paper, we propose a two-stage joint hovering altitude and power control solution for the resource allocation problem. Furthermore, Lagrange dual decomposition and concave-convex procedure (CCP) method are used to solve this problem. Finally, simulation results show the effectiveness of our proposed twostage joint optimization algorithm in terms of UAV network's total throughput.
\end{abstract}

Index Terms-Network association, cross-tier interference, heterogeneous networks, power control.

\section{INTRODUCTION}

Given the recent progress in the field of unmanned aerial vehicles (UAVs), it has become vitally important to bring drones into wireless communications considering their low cost, fast deployment, fully controllable mobility as well as the line of sight (LOS) communication links [1]. UAV communication networks along with traditional satellite networks and ground cellulars construct a space-air-ground three-tier heterogeneous network, which is capable of both providing seamless coverage as well as of further improving the channel capacity [2]. Due to spectrum scarcity, it is essential to sharing the spectrum among different kinds of communication subsystems. Particularly, the standard frequency of the fifth generation wireless systems $(5 \mathrm{G})$ is moving close to C-band and Ka-band, which are originally assigned to the airborne communication [3]. Hence, a well-implemented network association mechanism of spaceair-ground heterogeneous systems is beneficial in terms of both improving the resource utilization as well as of reducing the cross-tier interference.

As for the resource allocation problem in heterogeneous networks, Fooladivanda et al. in [4] investigated the user association and resource allocation in heterogeneous cellular networks in terms of orthogonal channel deployment, cochannel deployment as well as partially shared channel deployment. Furthermore, a distributed joint allocation algorithm is proposed for band selection and power allocation in order to maximize total capacity of a multi-mode and multi-band user terminal (MMT) by Choi et al. in [5]. Considering the aspect of energy efficiency, Xie et al. in [6] formulated an energy-efficient resource allocation problem as a Stackelberg game for heterogeneous cognitive femtocells. Furthermore, in [7], a mixed-integer programming problem was formulated for allocating subchannel and power resources in orthogonal frequency-division multiple access (OFDMA) hybrid networks with femtocells. However, these resource allocation mechanisms may not be suitable for the applications for the UAV aided space-air-ground heterogeneous network, because few of them considered the characteristics of UAVs in designing resource allocation algorithms, such as dynamic topology, flexible deployment, etc. Moreover, the resource allocation should take into account the inevitable cross-tier interference in space-air-ground hybrid networks [8].

Inspired by the above-mentioned open challenges, in this paper, we propose a two-stage joint hovering altitude and power control optimization for UAV networks in the context of a space-air-ground heterogeneous communication network considering diverse user's QoS requirements. Moreover, both the Lagrange dual decomposition and concave-convex procedure (CCP) method are used to approximatively solve the problem involved. Finally, extensive simulations show that our resource allocation mechanism is beneficial in terms of improving UAV network's total throughput considering the inevitable cross-tier interference.

The remainder of this article is outlined as follows. The system model and problem formulation are detailed in Section II. A two-stage joint hovering altitude and power control solution for UAV networks is elaborated in Section III. In Section IV, simulation results are provided for characterizing our proposed uplink resource allocation model for UAV networks, followed by our conclusions in Section V.

\section{System Model ANd Problem Formulation}

\section{A. System Model}

In this paper, as shown in Fig. 1, we consider a threetier hybrid network including a satellite network with a geosynchronous earth orbit satellite (GEO), a macrocell with a base station (MBS) and $M$ UAV networks sharing the same channel. Each UAV network is served by a hovering drone. Let $h_{m}$ represents the hovering altitude of the $m$-th drone. 


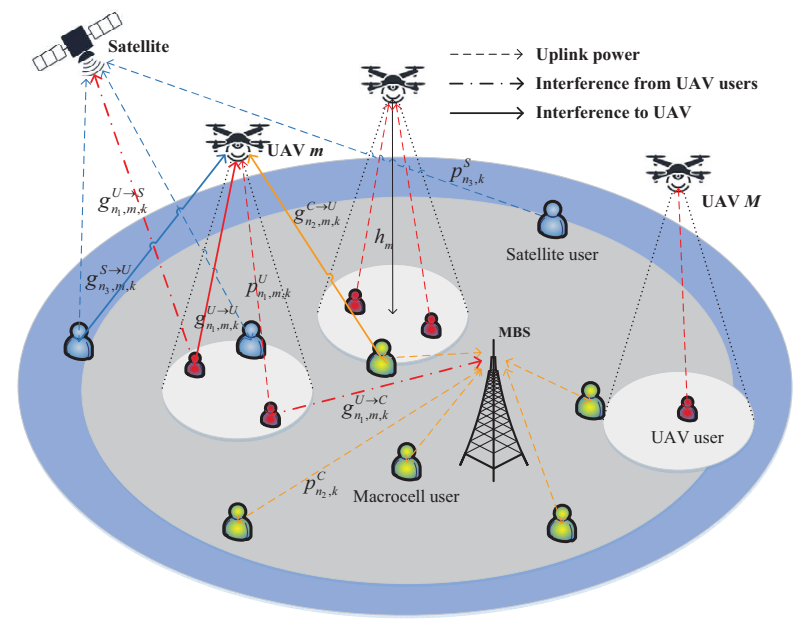

Fig. 1. The structure of satellite, UAV and macrocell three-tier hybrid network.

The coverage of $M$ UAV networks are overlaid within the coverage of the GEO as well as the macrocell. We focus our attention on the uplink power control of the users in the UAV networks. We assume that the uplink power of both satellite users and of macrocell users is equal.

The bandwidth of the channel is $B$, which is divided into $K$ subchannels. The channel fading between the MBS and users on the ground is the frequency-selective Reyleigh fading, while the communication channel between the hovering drone and users is dominated by the LoS path. The channel fading between the GEO and users on the ground is the Rician fading.

Let $N_{S}, N_{C}$ and $N_{U}$ denote the number of active users served by the GEO, the MBS and by the UAV, respectively. We assume that the satellite users and the macrocell users are uniformly distributed in each coverage area. In our model, two kinds of users with different QoS requirements are served in each UAV network. Specifically, the number of QoS-sensitive users requiring a high transmission rate of $R_{h}$ is $N_{u h}$, while the number of QoS-tolerant users with a low transmission rate requirement of $R_{l}$ is $N_{u l}$, where $N_{u h}+N_{u l}=N_{U}$. Let $\mathbb{N}_{u h}$ and $\mathbb{N}_{u l}$ represent the set of QoS-sensitive users and QoStolerant users, respectively. Then, we have $\left|\mathbb{N}_{u h}\right|=N_{u h}$ and $\left|\mathbb{N}_{u l}\right|=N_{u l}$, and $\mathbb{N}_{u h} \bigcap \mathbb{N}_{u l}=\emptyset$.

Let $g_{n_{1}, m, k}^{U \rightarrow S}, g_{n_{1}, m, k}^{U \rightarrow C}$ and $g_{n_{1}, m, k}^{U \rightarrow U}$ denote the channel gains on $k$-th subchannel from user $n_{1}$ in $m$-th UAV network to the GEO, to the MBS and to the hovering drone, respectively, where $n_{1} \in\left\{1,2, \ldots, N_{U}\right\}, m \in\{1,2, \ldots, M\}$ and $k \in\{1,2, \ldots, K\}$. In our model, $g_{n_{1}, m, k}^{U \rightarrow S}$ can be viewed as a constant because the UAV users locate far away from the GEO satellite, while $g_{n_{1}, m, k}^{U \rightarrow C}$ depends the channel state and the distance between each UAV user and the MBS. For the sake of analysis, we assume that the service radius of each drone can be neglected compared with its altitude, and hence $g_{n_{1}, m, k}^{U \rightarrow U}$ is only sensitive to the hovering altitude $h_{m}$ of the $m$-th drone, which can be formulated as:

$$
g_{n_{1}, m, k}^{U \rightarrow U}=\frac{\kappa}{h_{m}^{2}},
$$

where $\kappa$ denotes the unit power gain in terms of the reference distance $h_{r}=1 \mathrm{~m}$. Furthermore, let $g_{n_{2}, m, k}^{C \rightarrow U}$ represent the channel gain on $k$-th subchannel from user $n_{2}$ in the macrocell to the $m$-th hovering drone, while $g_{n_{3}, m, k}^{S \rightarrow U}$ denotes the channel gain on $k$-th subchannel from user $n_{3}$ in the satellite network to the $m$-th hovering drone, where $n_{2} \in\left\{1,2, \ldots, N_{C}\right\}$ and $n_{3} \in\left\{1,2, \ldots, N_{S}\right\}$. Moreover, let $p_{n_{2}, k}^{C}$ and $p_{n_{3}, k}^{S}$ represent the uplink transmission power of user $n_{2}$ in the macrocell and of user $n_{3}$ in the satellite network on $k$-th subchannel, respectively, while $p_{n_{1}, m, k}^{U}$ is the uplink transmission power of user $n_{1}$ in the $m$-th UAV network on $k$-th subchannel. In our model, we define $\mathbf{P}_{N_{U} \times M \times K}$ as the power allocation matrix for the users served by total $M$ UAV networks, and we have $[\mathbf{P}]_{n_{1}, m, k}=p_{n_{1}, m, k}^{U}$.

Here, we define a channel indicator matrix as $\mathbf{A}_{N_{U} \times M \times K}$, where $[\mathbf{A}]_{n_{1}, m, k}=a_{n_{1}, m, k}$. To elaborate, $a_{n_{1}, m, k}=1$ represents that the $k$-th subchannel is occupied by user $n_{1}$ in the $m$-UAV network, otherwise, $a_{n_{1}, m, k}=0$. We consider the additive white Gaussian noise (AWGN) with the variance of $\sigma^{2}$. Hence, as for the $m$-th UAV network, the received signalto-interference-plus-noise ratio (SINR) of the hovering drone from user $n_{1}$ accessing the $k$-th subchannel can be calculated by:

$$
\gamma_{n_{1}, m, k}=\frac{p_{n_{1}, m, k}^{U} g_{n_{1}, m, k}^{U \rightarrow U}}{g_{n_{2}, m, k}^{C \rightarrow U} p_{n_{2}, k}^{C}+g_{n_{3}, m, k}^{S \rightarrow U} p_{n_{3}, k}^{S}+\sigma^{2}},
$$

where $g_{n_{2}, m, k}^{C \rightarrow U} p_{n_{2}, k}^{C}$ is the interference from the user in the macrocell sharing the same sub-channel, while $g_{n_{3}, m, k}^{S \rightarrow U} p_{n_{3}, k}^{S}$ is the interference caused by the user in the satellite network occupying the $k$-th sub-channel. Remarkably, at most one user is capable of accessing the same subchannel at one moment in the macrocell, in the satellite network as well as in a UAV network. The co-interference between different UAV networks is negligible compared with the cross-tier interference from the macrocell and the satellite network.

Relying on the Shannon formula [9], the uplink capacity of $m$-th UAV network from its user $n_{1}$ on $k$-th subchannel can be calculated by:

$$
C_{n_{1}, m, k}=\frac{B}{K} \log _{2}\left(1+\gamma_{n_{1}, m, k}\right) .
$$

\section{B. Problem Formulation}

In this subsection, we will formulate the uplink resource allocation problem for the UAV network. Furthermore, we assume that the channel state information (CSI) as well as the result of uplink resource allocation can be forwarded to the users by the hovering drone based on the channel reciprocity. The total capacity of $M$ UAV networks can be given by:

$$
C_{\text {total }}=\sum_{m=1}^{M} \sum_{n_{1}=1}^{N_{U}} \sum_{k=1}^{K} a_{n_{1}, m, k} C_{n_{1}, m, k} .
$$

Hence, the uplink resource allocation problem can be for- 
mulated as:

$$
\begin{aligned}
& \max _{\left\{a_{n_{1}, m, k}, p_{n_{1}, m, k}, h_{m}\right\}} \sum_{m=1}^{M} \sum_{n_{1}=1}^{N_{U}} \sum_{k=1}^{K} a_{n_{1}, m, k} C_{n_{1}, m, k} \\
& \text { s.t. } \quad \text { (5a) : } \sum_{k=1}^{K} a_{n_{1}, m, k} p_{n_{1}, m, k}^{U} \leq P_{\max }^{U}, \quad \forall n_{1}, m, \\
& \text { (5b) : } p_{n_{1}, m, k}^{U} \geq 0, \quad \forall n_{1}, m, k, \\
& \text { (5c) : } \sum_{i, j \in \mathbb{M}, i \neq j}\left(h_{i}-h_{j}\right)^{2} \geq \chi^{2}, \\
& \text { (5d) }: h_{\min } \leq h_{m} \leq h_{\max }, \quad \forall m, \\
& \text { (5e) : } \sum_{k=1}^{K} a_{n_{u h}, m, k} C_{n_{u h}, m, k} \geq R_{h}, \quad \forall n_{u h}, m, \\
& \text { (5f) : } \sum_{k=1}^{K} a_{n_{u l}, m, k} C_{n_{u l}, m, k} \geq R_{l}, \quad \forall n_{u l}, m, \\
& \text { (5g) : } \sum_{m=1}^{M} \sum_{n_{1}=1}^{N_{U}} a_{n_{1}, m, k} p_{n_{1}, m, k}^{U} g_{n_{1}, m, k}^{U \rightarrow C} \leq I_{k}^{C}, \quad \forall k, \\
& \text { (5h): } \sum_{m=1}^{M} \sum_{n_{1}=1}^{N_{U}} a_{n_{1}, m, k} p_{n_{1}, m, k}^{U} g_{n_{1}, m, k}^{U \rightarrow S} \leq I_{k}^{S}, \quad \forall k \\
& \text { (5i) : } \sum_{n_{1}=1}^{N_{U}} a_{n_{1}, m, k} \leq 1, \quad \forall m, k, \\
& \text { (5j) : } a_{n_{1}, m, k} \in\{0,1\}, \quad \forall n_{1}, m, k,
\end{aligned}
$$

where $h_{\min }$ and $h_{\max }$ are the range of UAV's hovering altitude and $\chi^{2}$ is the minimal variance of the altitude of $M$ drones for safety flight and hovering, while $I_{k}^{C}$ and $I_{k}^{S}$ denotes the threshold of the interference from UAV networks on the $k$-th subchannel to the macrocell and the satellite network, respectively. The transmission rate requirement of QoS-sensitive and QoS-tolerant users is represented by $R_{h}$ and $R_{l}$, respectively. To elaborate further, (5a) and (5b) are users' power constraints, while $(5 \mathrm{c})$ and $(5 \mathrm{~d})$ are hovering altitude constraints. As for the QoS constraints (5e) and (5f), considering the QoS-tolerant users with a low transmission rate requirement of $R_{l}$, where $0<R_{l} \ll R_{h}$, hence we can neglect the constraint (5f) in problem (5) without loss of generality. Furthermore, $(5 \mathrm{~g})$ and $(5 \mathrm{~h})$ are interference constraint from macrocell and satellite network, respectively. Finally, the subchannel allocation constraints are given by $(5 \mathrm{i})$ and $(5 \mathrm{j})$.

\section{Two-Stage Joint Hovering Altitude And Power CONTROL SOLUTION}

In this section, we propose a two-stage joint optimization algorithm for our uplink resource allocation problem.

\section{A. Stage 1: Joint Subchannel and Power Control}

In the following, we study the joint subchannel and power control problem with given hovering altitude, where the initial $h_{m}^{0}$ constitutes a arithmetic progression ranging from $h_{\min }$ to $h_{\max }$. First of all, we relax the inter programming constraint $a_{n_{1}, m, k} \in\{0,1\}$ in $(5 \mathrm{j})$ to a continuous convex constraint $a_{n_{1}, m, k} \in[0,1]$.
Furthermore, let us introduce the auxiliary variable $\rho_{n_{1}, m, k}=a_{n_{1}, m, k} p_{n_{1}, m, k}^{U}$, and hence the uplink capacity of Eq. (3) can be converted to:

$$
\begin{aligned}
& \hat{C}_{n_{1}, m, k}= \\
& \frac{B}{K} \log _{2}\left(1+\frac{\rho_{n_{1}, m, k} g_{n_{1}, m, k}^{U \rightarrow U}}{a_{n_{1}, m, k}\left(g_{n_{2}, m, k}^{C \rightarrow U} p_{n_{2}, k}^{C}+g_{n_{3}, m, k}^{S \rightarrow U} p_{n_{3}, k}^{S}+\sigma^{2}\right)}\right),
\end{aligned}
$$

where $g_{n_{1}, m, k}^{U \rightarrow U}=\frac{\kappa}{h_{m}^{2}}$ and $h_{m} \triangleq h_{m}^{0}, m \in \mathbb{M}$.

Thus, we can obtain that our optimization objective $a_{n_{1}, m, k} \hat{C}_{n_{1}, m, k}$ is concave in $\left(a_{n_{1}, m, k}, \rho_{n_{1}, m, k}\right)$, based on which our joint subchannel and power control problem in Stage 1 can be reformulated as:

$$
\begin{aligned}
& \max _{\left\{a_{n_{1}, m, k}, \rho_{n_{1}, m, k}\right\}} \sum_{m=1}^{M} \sum_{n_{1}=1}^{N_{U}} \sum_{k=1}^{K} a_{n_{1}, m, k} \hat{C}_{n_{1}, m, k} \\
& \text { s.t. } \quad(7 \mathrm{a}): \sum_{k=1}^{K} \rho_{n_{1}, m, k} \leq P_{\max }^{U}, \quad \forall n_{1}, m, \\
& \text { (7b) : } \rho_{n_{1}, m, k} \geq 0, \quad \forall n_{1}, m, k, \\
& \text { (7c) : } \sum_{k=1}^{K} a_{n_{u h}, m, k} \hat{C}_{n_{u h}, m, k} \geq R_{h}, \quad \forall n_{u h}, m, \\
& \text { (7d) }: \sum_{m=1}^{M} \sum_{n_{1}=1}^{N_{U}} \rho_{n_{1}, m, k} g_{n_{1}, m, k}^{U \rightarrow C} \leq I_{k}^{C}, \quad \forall k, \\
& \text { (7e) : } \sum_{m=1}^{M} \sum_{n_{1}=1}^{N_{U}} \rho_{n_{1}, m, k} g_{n_{1}, m, k}^{U \rightarrow S} \leq I_{k}^{S}, \quad \forall k, \\
& \text { (7f) : } \sum_{n_{1}=1}^{N_{U}} a_{n_{1}, m, k} \leq 1, \quad \forall m, k, \\
& \text { (7g) : } a_{n_{1}, m, k} \in[0,1], \quad \forall n_{1}, m, k .
\end{aligned}
$$

Obviously, our joint subchannel and power control problem in (7) is a convex optimization problem. Hence, we can use the Lagrangian dual decomposition method to solve our joint subchannel and power control problem in (7). By neglecting mathematical derivations, the optimal solution of the power allocation $p_{n_{1}, m, k}^{U *}=\rho_{n_{1}, m, k}^{*} / a_{n_{1}, m, k}$ in $m$-th UAV network on the $k$-th subchannel for user $n_{1}$ can be given by:

$$
p_{n_{1}, m, k}^{U *}=\left\{\begin{array}{l}
\max \left\{0, \frac{B\left(1+\mu_{i, m}\right)}{K \ln 2 \times \Theta_{n_{1}}}-\frac{\Delta}{g_{j, m, k}^{U \rightarrow U}}\right\}, n_{1} \in \mathbb{N}_{u h}, \\
\max \left\{0, \frac{B}{K \ln 2 \times \Theta_{n_{1}}}-\frac{\Delta}{g_{j, m, k}^{U \rightarrow U}}\right\}, n_{1} \in \mathbb{N}_{u l},
\end{array}\right.
$$

where $\Delta=g_{n_{2}, m, k}^{C \rightarrow U} p_{n_{2}, k}^{C}+g_{n_{3}, m, k}^{S \rightarrow U} p_{n_{3}, k}^{S}+\sigma^{2}$ and $\Theta_{n_{1}} \stackrel{\text { (8) }}{=}$ $\lambda_{n_{1}, m}+\nu_{k} g_{n_{1}, m, k}^{U \rightarrow C}+\omega_{k} g_{n_{1}, m, k}^{U \rightarrow S}, n_{1} \in\left\{1,2, \ldots, N_{U}\right\}$. Similarly, considering $a_{n_{1}, m, k}^{*} \in[0,1]$ in $(7 \mathrm{~g})$, the optimal solution $a_{n_{1}, m, k}^{*}, \forall n_{1}, m, k$ are given by:

$$
\left\{\begin{array}{l}
a_{n_{1}, m, k}^{*}=0 \quad \text { and }\left.\quad \frac{\partial \boldsymbol{\Phi}}{\partial a_{n_{1}, m, k}}\right|_{a_{n_{1}, m, k}=0}<0 \\
a_{n_{1}, m, k}^{*} \in(0,1) \text { and }\left.\frac{\partial \boldsymbol{\Phi}}{\partial a_{n_{1}, m, k}}\right|_{a_{n_{1}, m, k}=a_{n_{1}, m, k}^{*}}=0 \\
a_{n_{1}, m, k}^{*}=1 \quad \text { and }\left.\quad \frac{\partial \boldsymbol{\Phi}}{\partial a_{n_{1}, m, k}}\right|_{a_{n_{1}, m, k}=1}>0
\end{array}\right.
$$


where for the QoS-sensitive user $i \in \mathbb{N}_{u h}$,

$$
\begin{aligned}
& \frac{\partial \boldsymbol{\Phi}}{\partial a_{i, m, k}}=\left(1+\mu_{i, m}\right) \frac{B}{K} \log _{2}\left(1+\frac{p_{i, m, k}^{U *} g_{i, m, k}^{U \rightarrow U}}{\Delta}\right) \\
& -\left(1+\mu_{i, m}\right) \frac{B p_{i, m, k}^{U *} g_{i, m, k}^{U \rightarrow U}}{K \ln 2 \times\left(\Delta+p_{i, m, k}^{U *} g_{i, m, k}^{U \rightarrow U}\right)}-\lambda_{i, m} p_{i, m, k}^{U *} \\
& -\nu_{k} p_{i, m, k}^{U *} g_{i, m, k}^{U \rightarrow C}-\omega_{k} p_{i, m, k}^{U *} g_{i, m, k}^{U \rightarrow S}-\xi_{m, k},
\end{aligned}
$$

and for the QoS-tolerant user $j \in \mathbb{N}_{u l}$, we have:

$$
\begin{aligned}
\frac{\partial \Phi}{\partial a_{j, m, k}} & =\frac{B}{K} \log _{2}\left(1+\frac{p_{j, m, k}^{U *} g_{j, m, k}^{U \rightarrow U}}{\Delta}\right) \\
& -\frac{B p_{j, m, k}^{U *} g_{j, m, k}^{U \rightarrow U}}{K \ln 2 \times\left(\Delta+p_{j, m, k}^{U *} g_{j, m}^{U \rightarrow,}\right)}-\lambda_{j, m} p_{j, m, k}^{U *} \\
& -\nu_{k} p_{j, m, k}^{U *} g_{j, m, k}^{U \rightarrow C}-\omega_{k} p_{j, m, k}^{U *} g_{j, m, k}^{U \rightarrow S}-\xi_{m, k},
\end{aligned}
$$

where $\lambda, \mu, \nu, \omega$ and $\boldsymbol{\xi}$ are the Lagrangian multipliers.

In our model, at most one user is allowed to access the same subchannel at one moment in a UAV network, and we have:

$$
n_{1}^{*}=\arg \max _{n_{1}} \frac{\partial \Phi}{\partial a_{n_{1}, m, k}}, \quad \forall m, k,
$$

where $a_{n_{1}^{*}, m, k}^{*}=1$ represents the suboptimal channel indicator variable. Moreover, we can use the subgradient method to update the Lagrangian multipliers $\boldsymbol{\lambda}, \boldsymbol{\mu}, \boldsymbol{\nu}, \boldsymbol{\omega}$ and $\boldsymbol{\xi}$. Hence, we can obtain the optimal solution $\left\{a_{n_{1}^{*}, m, k}^{*}, p_{n_{1}, m, k}^{U *}\right\}$ of joint subchannel and power control for each users in UAV networks considering a fixed deployment altitude of hovering drones.

\section{B. Stage 2: Hovering Altitude Optimization}

In Stage 1, we fix the deployment altitude of each hovering drone and search for the optimal joint subchannel and power control mechanism for each user in UAV network, denoted as $\left\{a_{n_{1}^{*}, m, k}^{*}, p_{n_{1}, m, k}^{U *}\right\}$, where $n_{1} \in\left\{1,2, \ldots, N_{U}\right\}$, $m \in\{1,2, \ldots, M\}$ and $k \in\{1,2, \ldots, K\}$. In the following, we try to determine the optimal hovering altitude of each drone based on the results obtained from Stage 1. Considering the safety hovering altitude constraints of $(5 \mathrm{c})$ and $(5 \mathrm{~d})$ in our original problem formulation in (5), we have:

$$
\begin{aligned}
& \max _{\left\{h_{m}\right\}} \sum_{m=1}^{M} \sum_{n_{1}=1}^{N_{U}} \sum_{k=1}^{K} a_{n_{1}^{*}, m, k}^{*} \frac{B}{K} \log _{2}\left(1+\frac{\kappa p_{n_{1}, m, k}^{U *}}{h_{m}^{2} \Delta}\right) \\
& \text { s.t. } \quad(13 \mathrm{a}): \sum_{i, j \in \mathbb{M}, i \neq j}\left(h_{i}-h_{j}\right)^{2} \geq \chi^{2} \\
& \quad(13 \mathrm{~b}): h_{m} \leq h_{\max }, \quad \forall m, \\
& (13 \mathrm{c}): h_{m} \geq h_{\min }, \quad \forall m .
\end{aligned}
$$

The hovering altitude optimization problem in (13) can be reformulated as a difference of convex (DC) programming, which can be given by:

$$
\begin{array}{ll}
\min _{\mathbf{h}} \quad 0-g_{0}(\mathbf{h}) \\
\text { s.t. } \quad(14 \mathrm{a}): \chi^{2}-g_{1}(\mathbf{h}) \leq 0, \\
& (14 \mathrm{~b}): h_{m} \leq h_{\max }, \quad \forall m, \\
& (14 \mathrm{c}): h_{m} \geq h_{\min }, \quad \forall m .
\end{array}
$$

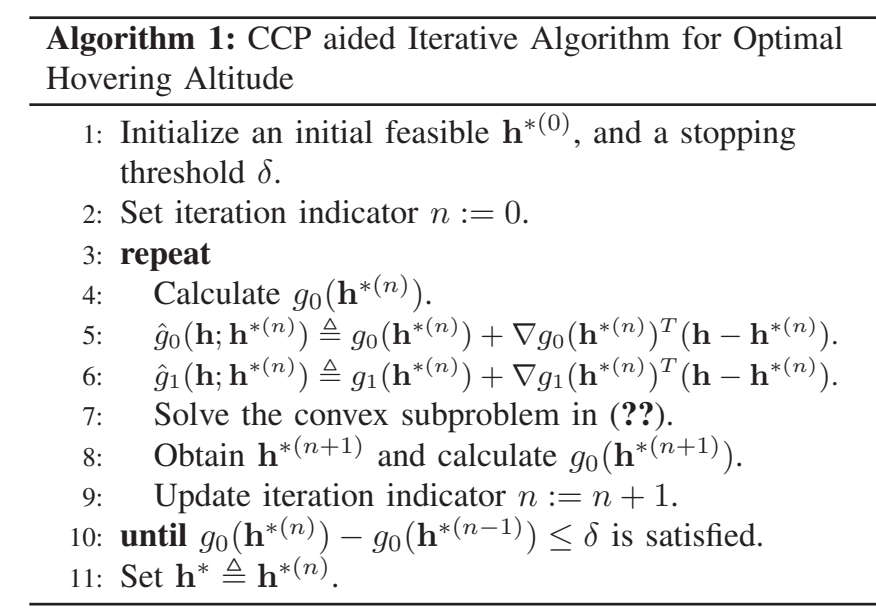

where the objective function can be expressed as:

$$
g_{0}(\mathbf{h})=\sum_{m=1}^{M} \sum_{n_{1}=1}^{N_{U}} \sum_{k=1}^{K} a_{n_{1}^{*}, m, k}^{*} \frac{B}{K} \log _{2}\left(1+\frac{\kappa p_{n_{1}, m, k}^{U *}}{h_{m}^{2} \Delta}\right),
$$

and $g_{1}(\mathbf{h})$ can be given by:

$$
g_{1}(\mathbf{h})=\sum_{i, j \in \mathbb{M}, i \neq j}\left(h_{i}-h_{j}\right)^{2} .
$$

Specifically, $g_{1}(\mathbf{h})$ is a quadratic form, which can be rewritten as $g_{1}(\mathbf{h})=\mathbf{h}^{T} \mathbf{Q h}$, where $\mathbf{Q}=\operatorname{diag}(M)-\mathbf{1}$. Moreover, $\operatorname{diag}(M)$ denotes a diagonal matrix with all diagonal elements equaling $M$ and $\mathbf{1}$ is an $M \times M$ matrix with all elements being 1 . Hence, both $g_{0}(\mathbf{h})$ and $g_{1}(\mathbf{h})$ in (14) are convex functions. Hence, we can use the CCP method to solve the problem in (14), where we are capable of achieving the locally optimal result of the non-convex problem through solving a series of iterative convex subproblems as shown in Algorithm. 1.

Hence, relying on the CCP aided iterative algorithm, given fixed $\left\{a_{n_{1}^{*}, m, k}^{*}, p_{n_{1}, m, k}^{U *}\right\}$, we obtain the optimal hovering altitude vector represented by $\mathbf{h}^{*}$. Thus, the total capacity of UAV networks can be recalculated as $C_{\text {total }}\left(a_{n_{1}^{*}, m, k}^{*}, p_{n_{1}, m, k}^{U *}, \mathbf{h}^{*}\right)$. Relying on the aforementioned two iterative stages, and letting $\Lambda$ be the stopping threshold of our two-stage resource allocation scheme, if the following condition is satisfied:

$C_{\text {total }}^{(i+1)}\left(a_{n_{1}^{*}, m, k}^{*}, p_{n_{1}, m, k}^{U *}, \mathbf{h}^{*}\right)-C_{\text {total }}^{(i)}\left(a_{n_{1}^{*}, m, k}^{*}, p_{n_{1}, m, k}^{U *}, \mathbf{h}^{*}\right) \leq \Lambda$, the final optimal uplink total capacity of $M$ UAV networks can be given by:

$$
C_{\text {total }}^{*} \triangleq C_{\text {total }}^{(i+1)}\left(a_{n_{1}^{*}, m, k}^{*}, p_{n_{1}, m, k}^{U *}, \mathbf{h}^{*}\right),
$$

where the optimal subchannel and power control result is given by $\left\{a_{n_{1}^{*}, m, k}^{*}, p_{n_{1}, m, k}^{U *}\right\} \triangleq\left\{a_{n_{1}^{*}, m, k}^{*}, p_{n_{1}, m, k}^{U *}\right\}^{(i+1)}$ as well as the optimal hovering altitude $\mathbf{h}^{*} \triangleq \mathbf{h}^{*(i+1)}$.

\section{Simulation Results}

In our simulation, three kinds of users are located in a $500 \mathrm{~m} \times 500 \mathrm{~m}$ square region. $N_{C}=10$ macrocell users and $N_{S}=10$ satellite users are randomly distributed in the area. Moreover, the coverage radius of each drone is $50 \mathrm{~m}$ and UAV users are randomly distributed in each coverage area. The 


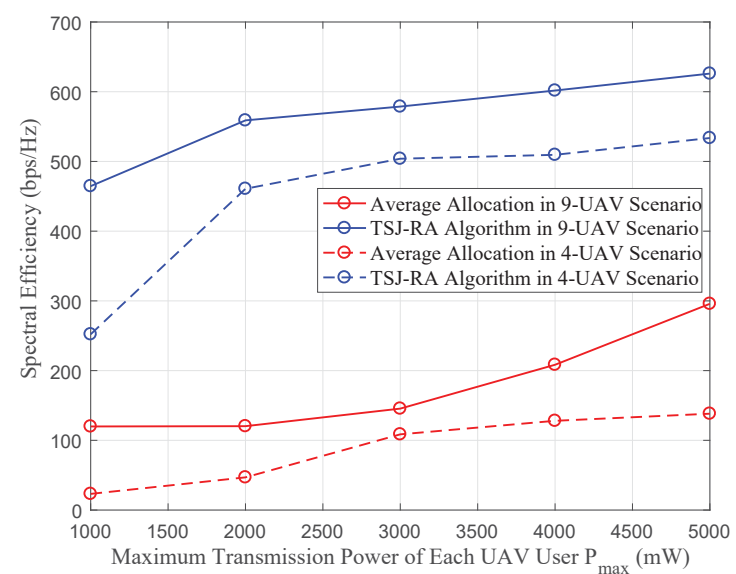

Fig. 2. Spectrum efficiency versus maximum transmission power of UAV users parameterized by different number of UAV networks.

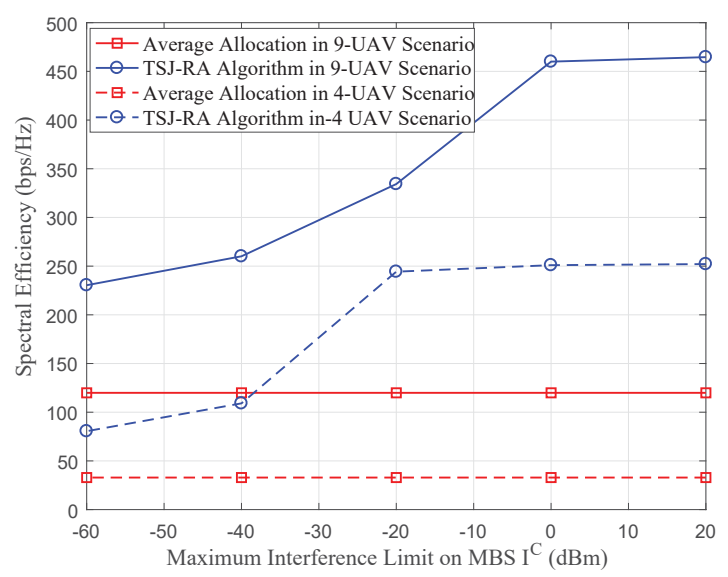

Fig. 3. Spectrum efficiency versus maximum interference limit of the MBS parameterized by different number of UAV networks.

altitude of GEO is $36000 \mathrm{~km}$ and only one MBS is considered in the simulation. The carrier frequency is $2.4 \mathrm{GHz}$ and the total number of the subchannel is $K=128$, each of which has a bandwidth of $15 \mathrm{kHz}$. The AWGN power spectrum density is $-174 \mathrm{dBm} / \mathrm{Hz}$. Furthermore, the channel between users and the MBS follows Rayleigh fading. By contrast, the channels between users and UAVs and the GEO follow Rician fading with $5 \mathrm{~dB}$ Rician factor. Let the reference-distance unit power gain be $\kappa=1.4 \times 10^{-4}[10]$. The hovering altitude of drones spans from $200 \mathrm{~m}$ to $400 \mathrm{~m}$. Our proposed UAV hovering altitude aided resource allocation mechanism is denoted as "TSJ-RA" in our simulation.

In the following, we consider two scenarios with 4 UAVs and 9 UAVs, respectively. Each drone serves $N_{U}=4$ UAV users. In the 4-UAV scenario, there are total 8 QoS-sensitive users and 8 QoS-tolerant users, while there are total 24 QoS-sensitive users and 12 QoS-tolerant users in the 9-UAV scenario. Moreover, the minimum data rate requirement of

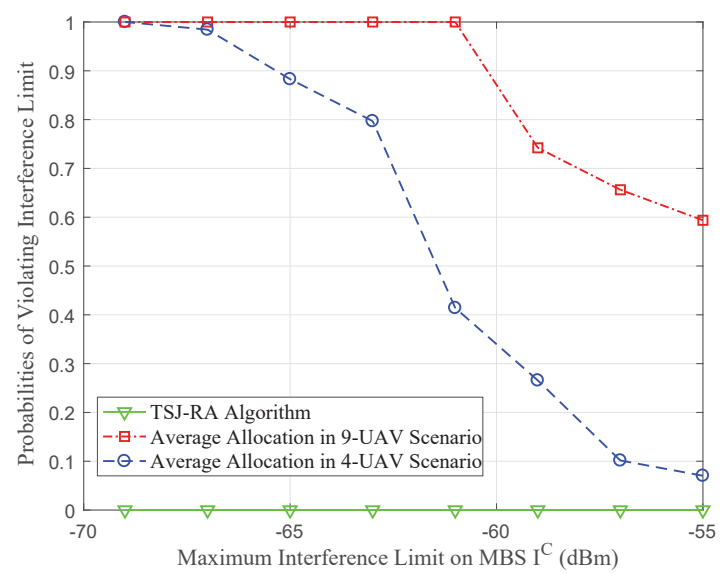

Fig. 4. Probabilities of violating the maximum interference limit on the MBS.

QoS-sensitive users is $R_{h}=30 \mathrm{kbps}$. We define the spectrum efficiency (SE) of UAV networks to evaluate the effectiveness of our proposed algorithm as: $\mathrm{SE}=C_{\text {total }} / B \quad(\mathrm{bps} / \mathrm{Hz})$.

Fig. 2 shows the impact of the maximum transmission power $p_{\max }^{U}$ on the UAV network's SE, where the maximum interference limit of both the MBS and the GEO is $0 \mathrm{dBm}$, i.e. $I^{C}=0 \mathrm{dBm}$ and $I^{S}=0 \mathrm{dBm}$ for all subchannels $k \in\{1,2, \ldots, K\}$. It can be observed that our proposed TSJ-RA algorithm outperforms the average resource allocation scheme $^{1}$ in terms of the SE. It is because the proposed TSJRA algorithm jointly optimizes the altitudes of the drones and transmission power of all users, achieving a decent SE performance and satisfying all the constraints all the time. As a comparison, the comparison algorithm is not aware of system configuration and introduces significant SE loss. Besides, higher SE is obtained with a loose transmission power constraint. Meanwhile, a dense UAV deployment is capable of substantially increasing the network's SE.

Fig. 3 demonstrates the performance of UAV network's SE characterized by the maximum interference limit of the MBS, i.e. $I^{C}$, with respect to $P_{\max }^{U}=1000 \mathrm{~mW}$ and $I^{S}=0 \mathrm{dBm}$. Since the average resource allocation scheme does not rely on the interference limit, the spectrum efficiency is not improved with the increase of MBS's interference limit. As for the TSJRA algorithm, a loose interference limit on the MBS yields a high SE of UAV networks to some extent. It is because that with a loose interference limit, UAV users are capable of using higher transmission power, while with a strict interference limit, UAV users have to properly decrease the transmission power to satisfy the preset constraint. Besides, it can seen that when the interference limit is loose enough, such as $I^{C}=0 \mathrm{dBm}$ for 9-UAV scenario and $I^{C}=-20 \mathrm{dBm}$ for 4 -UAV scenario, the $\mathrm{SE}$ remains unchanged. It is because with a loose threshold,

${ }^{1}$ In this paper, the average resource allocation scheme means that subchannels as well as power are uniformly allocated to two kinks of users without considering the interference limit of the MBS and the GEO under the constraint of a secure hovering altitude of each drone. 


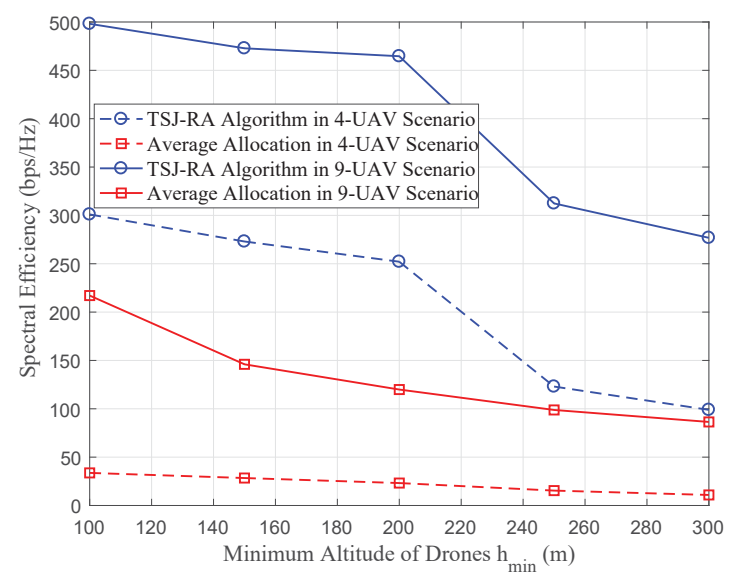

Fig. 5. Spectrum efficiency versus minimum hovering altitude parameterized by different number of UAV networks.

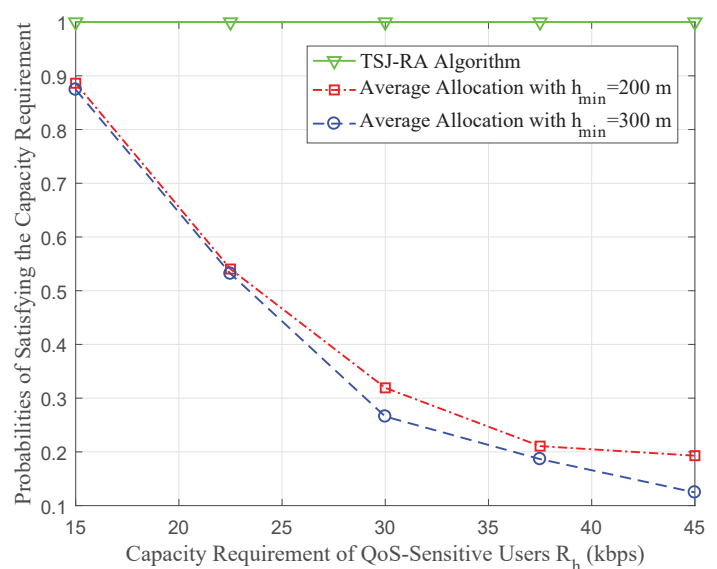

Fig. 6. Probabilities of satisfying capacity requirement of QoS-sensitive users in terms of different minimum hovering altitude of drones.

the pre-set interference constraint can be always satisfied with the given maximum available transmission power. To elaborate a little further, Fig. 4 portrays the probabilities of violating the maximum interference limit on the MBS, which is defined as the ratio of the number of subchannels with interference higher than pre-set maximum limit to the total number of subchannels. We can conclude that our proposed algorithm satisfy the interference limit for all subchannels $k \in\{1,2, \ldots, K\}$ at all given $I^{C}$ values. However, the average algorithm has a high probability of violating the interference limit when the interference requirement is stringent.

In Fig. 5, we evaluate the performance of $\mathrm{SE}$ versus different minimum hovering altitudes in different scenarios. It can be seen that a lower minimum hovering altitude is beneficial in terms of improving the SE of the total UAV networks relying on both our proposed TSJ-RA algorithm as well as on the average algorithm. Furthermore, Fig. 6 demonstrates the probabilities of satisfying the pre-set capacity requirement for QoS-sensitive users versus different values of $R_{h}$, which is defined as the ratio of the number of QoSsensitive users with satisfied capacity to the total number of QoS-sensitive users. It can be seen that our proposed algorithm always outperforms the comparison algorithm at all given $R_{h}$ values. It is because that our the proposed algorithm considers the capacity requirement of QoS-sensitive users, making the QoS-sensitive users have high priorities to obtain the channels. As a result, the probability of satisfying capacity requirement for QoS-sensitive users equals to 1 all the time. By contrast, the average allocation is not aware of the pre-set capacity constraint. Especially when the capacity requirement is stringent, i.e. $40 \mathrm{kbps}$, only around $10 \%$ of QoS-sensitive users can achieve decent capacity higher than the pre-set constraint.

\section{CONCLUSIONS}

In this paper, we formulated a two-stage joint hovering altitude and power control for UAV networks considering the feasible deployment of drones in the context of a space-airground three-tier heterogeneous network. We used Lagrange dual decomposition and CCP method to provide a near optimal solution for our proposed problem. Extensive simulations were conducted in order to show the performance of our network association mechanism, which yielded an improved UAV network's throughput.

\section{REFERENCES}

[1] J. Wang, C. Jiang, Z. Han, Y. Ren, R. G. Maunder, and L. Hanzo, "Taking drones to the next level: Cooperative distributed unmannedaerial-vehicular networks for small and mini drones," IEEE VehIcular Technology MagazIne, vol. 12, no. 3, pp. 73-82, Sept. 2017.

[2] M. Mozaffari, W. Saad, M. Bennis, and M. Debbah, "Efficient deployment of multiple unmanned aerial vehicles for optimal wireless coverage," IEEE Communications Letters, vol. 20, no. 8, pp. 1647-1650, Aug. 2016

[3] E. Lagunas, S. K. Sharma, S. Maleki, S. Chatzinotas, and B. Ottersten, "Resource allocation for cognitive satellite communications with incumbent terrestrial networks," IEEE Transactions on Cognitive Communications and Networking, vol. 1, no. 3, pp. 305-317.

[4] D. Fooladivanda and C. Rosenberg, "Joint resource allocation and user association for heterogeneous wireless cellular networks," IEEE Transactions on Wireless Communications, vol. 12, no. 1, pp. 248-257.

[5] Y. Choi, H. Kim, S.-w. Han, and Y. Han, "Joint resource allocation for parallel multi-radio access in heterogeneous wireless networks," IEEE Transactions on Wireless Communications, vol. 9, no. 11, pp. 3324 3329, Nov. 2010

[6] R. Xie, F. R. Yu, H. Ji, and Y. Li, "Energy-efficient resource allocation for heterogeneous cognitive radio networks with femtocells," IEEE Transactions on Wireless Communications, vol. 11, no. 11, pp. 39103920, Nov. 2012

[7] H. Zhang, C. Jiang, N. C. Beaulieu, X. Chu, X. Wen, and M. Tao, "Resource allocation in spectrum-sharing OFDMA femtocells with heterogeneous services," IEEE Transactions on Communications, vol. 62, no. 7, pp. 2366-2377, Jul. 2014.

[8] H. Wang, J. Wang, G. Ding, L. Wang, T. A. Tsiftsis, and P. K. Sharma, "Resource allocation for energy harvesting-powered D2D communication underlaying UAV-assisted networks," IEEE Transactions on Green Communications and Networking, Oct. 2017.

[9] C. E. Shannon, "A mathematical theory of communication," $A C M$ SIGMOBILE Mobile Computing and Communications Review, vol. 5, no. 1, pp. 3-55, Jan. 2001.

[10] H. He, S. Zhang, Y. Zeng, and R. Zhang, "Joint altitude and beamwidth optimization for UAV-enabled multiuser communications," IEEE Communications Letters, DOI:10.1109/LCOMM.2017.2772254, Nov. 2017. 